Fariñas, L. ', Badal Marín, E. ${ }^{2}$, Martínez Gimeno, M.A. ${ }^{3}$, Bonet, L. ${ }^{4}$, Manzano, J. ${ }^{5}$ (P)

1 Investigadora, Departamento Tecnología de Alimentos (UPV), maferfa@upv.es

\title{
A27-2020 $\quad{ }^{2}$ Ingeniero agrónomo, Servicio de Tecnología del Riego (IVIA), $\quad$ badal_edu@gva.es
}

DOI:10.31428/10317/8682

${ }^{4}$ Ingeniero agrónomo, Servicio de Tecnología del Riego (STR), Institut Valencià de Investigacions Agràries (IVIA), bonet_lui@gva.es

${ }^{5}$ Profesor, Universitat Politècnica de València, Centro Valenciano de Estudios sobre el Riego, juamanju@agf.upv.es

En este estudio se presentan los últimos datos experimentales en campo en hojas de Clemenules $x$ carrizo. Se comparan los datos tomados con la tradicional cámara de presión y con la novedosa técnica de Espectroscopía Ultrasónica Resonante mediante sensores sin contacto (NC-RUS). Las plantas fueron sometidas a tres tratamientos de riego: T100, plantas regadas al 100\% de la evapotranspiración de cultivo (ETc); T50, riego al 75\% de la ETc; y T25, riego al $50 \%$ de la ETc.

Palabras clave: Estrés hídrico, Sensores, Nuevas Tecnologías, Ultrasonidos, Cítricos

\section{Objetivos del proyecto CitRUS}

\section{PRINCIPAL}

\section{PARCIALES}

Estudiar la validez de la técnica NC-RUS como indicador del estado hídrico en plantas mediante medidas fisiológicas en el campo.

Aplicar en cítricos la técnica de NC-RUS en campo implementando un sistema portátil.

Correlacionar parámetros ultrasónicos de las hojas con parámetros de control del estado hídrico de la planta.

Correlacionar los parámetros ultrasónicos con dosis de riego.

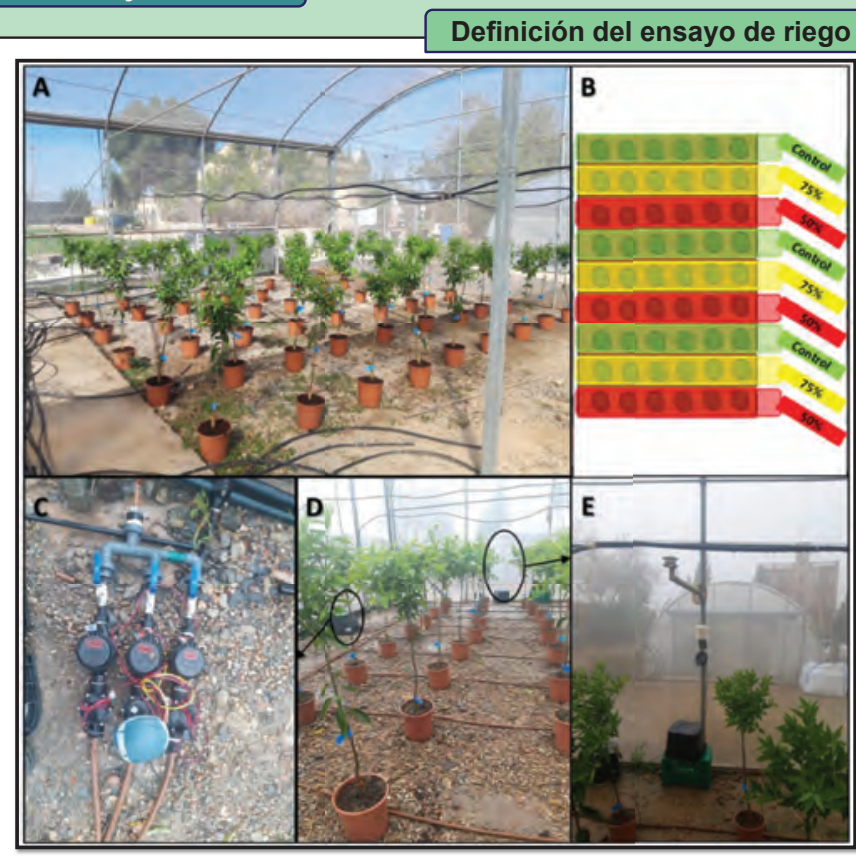

A) Parcela pre-montaje; B) Esquema de tratamientos de riego: C) Riego; D) Parcela postmontaje; E) Sensores de humedad relativa, temperatura y radiación

\section{MATERIAL:}

Parcela experimental UPV bajo malla de sombreo.

Plantones de Citrus clementina sobre patrón de Citrange Carrizo.

TRATAMIENTO DE RIEGO:

- 3 tratamientos de riego: $100 \%$ (T100), $75 \%$ (T50) y $50 \%$ (T25) sobre las necesidades de cultivo calculadas mediante el método FAO Penman-Monteith) con 3 repeticiones.

INSTALACIÓN DE RIEGO:

Riego localizado.

Ejecutado con emisores de $2 \mathrm{l} / \mathrm{h}$.

Automatizado mediante un programador.

POTENCIAL HÍDRICO DE TALLO AL MEDIODÍA SOLAR:

Mediante una cámara de presión: Model 600, PMS.

Sobre 12 hojas por tratamiento, elegidas aleatoriamente

\section{CONCLUSIONES}

La Espectroscopía Resonante Ultrasónica Sin Contacto (NC-RUS) ha demostrado ser una técnica que puede aplicarse en hojas de Clemenules en campo mediante la implementación de un sistema portátil.

Sin embargo, a lo largo de los experimentos, no pudieron observarse cambios significativos en el potencial hídrico de tallo que den cabida a correlacionar con los datos de frecuencia de resonancia provenientes de NC-RUS. Las plantas mantuvieron un estado hídrico adecuado debido a las condiciones climáticas que condicionaron una baja demanda evapotranspirativa del cultivo.

En consecuencia, se volverán a tomar medidas con ambas técnicas sobre los plantones el próximo verano, cuando se espera alcanzar niveles de estrés elevados.

A

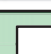

Espectroscopía Ultrasónica Resonante Sin Contacto (NC-RUS)

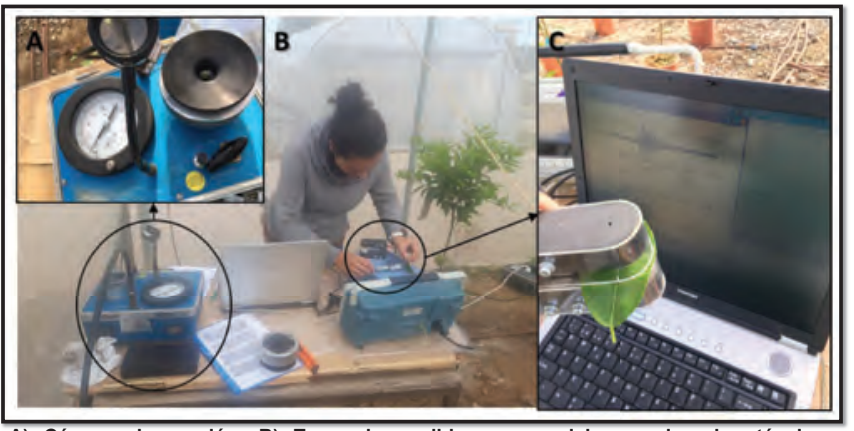

A) Cámara de presión; B) Toma de medidas en paralelo con las dos técnicas contempladas: C) Sistema portátil NC-RUS

EQUIPO DE ULTRASONIDOS

Dos transductores de ultrasonidos diseñados para su uso en aire (USBioMat, ITEFI-CSIC).

Rango de frecuencias de trabajo de los sensores/transductores ultrasónicos: 0.3 y $0.8 \mathrm{MHz}$

- Soporte para mantener los sensores enfrentados a $30 \mathrm{~mm}$ de distancia

- Excitación mediante pulser-reciever 5077PR, Olympus con pulso de semionda cuadrada de $400 \mathrm{~V}$.

D Digitalización de las señales mediante el osciloscopio DPO2002, Tektronix.

- Control de los dispositivos vía USB por un portátil donde también se guardaron las señales.

\section{PROCESAMIENTO:}

Filtrado de las señales (frecuencia de corte de $10 \mathrm{MHz}$ ).

Transformada de Fourier rápida sobre las señales recibidas sustrayendo el blanco de calibración y corrigiendo en ganancia (Numpy, Scipy y pyVISA, Python).

\section{Resultados y Discusión}

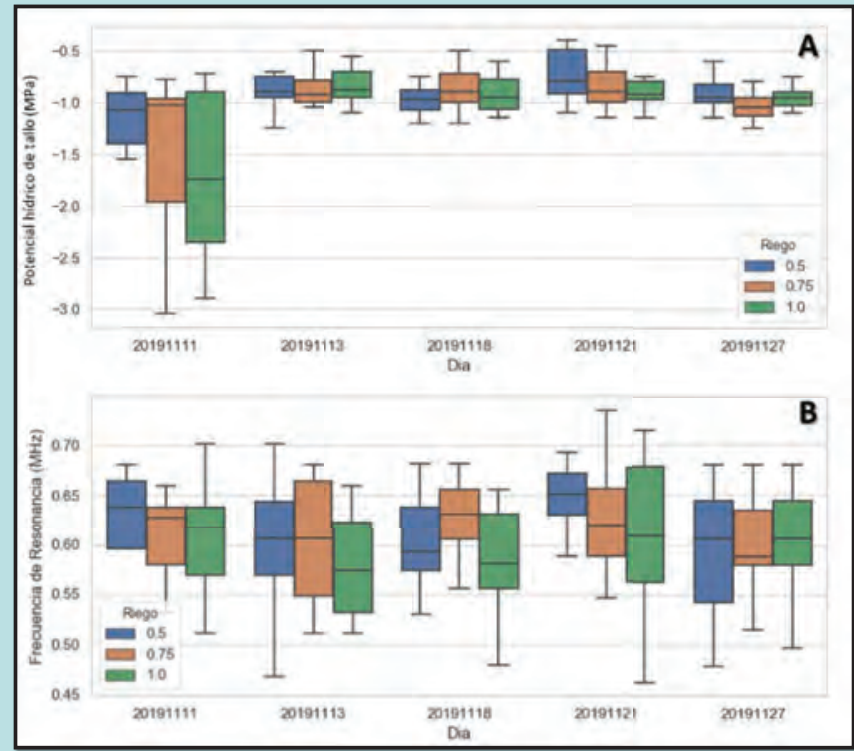

Valores medidos sobre hojas con diferente tratamiento de riego a lo largo de las jornadas de 5 jornas de experimentación a mediodía solar: A) potencial hídrico de tallo; B) frecuencia de resonancia 